\title{
Implementing virtual experiences and remote assessments during the COVID-19 pandemic: A college experience
}

\author{
M.N. Daftary, J. Jorden, M. Habib, I. Pather, T. Tofade* \\ Howard University College of Pharmacy, USA
}

\section{Keywords \\ Assessment \\ Proctoring \\ Telehealth \\ Experiential}

*Corresponding author:

toyin.tofade@howard.edu

\begin{abstract}
Summary: With nine weeks remaining in the term, COVID-19 necessitated emergency remote learning and remote online exams. This required a series of software trainings for administrators, faculty and students at Howard University College of Pharmacy, USA. The experiential department also had to look beyond normal clerkship placements. The described telemedicine and virtual experiences were used to supplement face-to-face experiences. Assessments for both learning innovations were successful, based on faculty and student feedback. Student reflections describe how COVID-19 helped advance the use of telehealth and indicate their learning of clinical decision-making skills, effective communication, and the digital health industry. A formal evaluation of outcomes from the implemented pandemic innovations is being planned. The use of online proctoring with artificial intelligence during the emergency remote learning was successful. With software training, this type of service can be adopted to ensure the integrity of assessments. The cost of the service and the reporting time should be considered in academic planning.
\end{abstract}

\section{Background and Context}

In March 2020, Howard University College of Pharmacy shifted to emergency remote learning due to the COVID-19 pandemic. This created a need for remote assessments with assured integrity. Despite having paperless assessments for several years, the provision of remote exams was new to the College. With nine weeks remaining in the University's term, faculty quickly researched several options: online assessments with no backward navigation, open-book exams with appropriate questions, videoconferencing, or use of digital proctoring systems that rely on a webcam and artificial intelligence (Al) to evaluate integrity. The choice of the latter came easily as the college was already using ExamSoft (paperless online assessment system). The following software was procured from the same vendor: ExamID (facial recognition), ExamMonitor (uses Al to monitor and flag suspicious activity even with a wifi disabled environment), and ProctorU (uses a person to review flagged video segments) (Examsoft ID verification, 2020; Examsoft Exam Monitor, 2020). These software systems utilise both a computer and webcam, a facial recognition algorithm and online proctoring supplemented by human review of flagged segments. Students were informed of these changes due to an emergency remote learning environment. Email instructions were distributed to students. Administrators and faculty who had existing courses were trained and available for one on one test-taker training.

Additionally, the experiential department was innovative and looked beyond the normal clerkship placement to ensure adequate experiential learning needed for graduation and licensure. The Accreditation Council for Pharmacy Education (ACPE) allowed specific pharmacy practice experiences to be completed by virtual/distance learning, thus enabling students to complete the Advanced Pharmacy Practice Experiences (APPE) year (ACPE, 2015). 


\section{Educational Description}

Students signed consent forms for the use of the software systems and for their digital image to be taken and stored within the system. Assessments used both ExamID and ExamMonitor. ProctorU uses human review of the flagged video segments which constitute a small section of the exam. Between March and May 2020, the College had 14 courses in progress for the spring 2020 term. Prior to 22nd March 2020, the College had 12 assessments completed via Examsoft. After 22nd March 2020 up until the end of the term, there were a total of 45 assessments in Examsoft, resulting in a total of 2,006 test-taking events which utilised ExamMonitor.

The recordings were returned to the College by ExamMonitor after review by ProctorU, and the flagged incidents were reported. Faculty categorised them into categories: no presumption of breach, possibility of an academic integrity issue, and high likelihood of academic integrity breach. Course coordinators reviewed the flagged video segments to decide if cheating had occurred. A total of 523 incidents were reported to the College. Course coordinators reviewed the flags and then created a course level summary. The faculty were encouraged to resolve the incident with the student. If resolution was not possible, the issue was referred to the Department chair.

Both both qualitative and quantitative feedback was received. Quantitative data were provided by Examsoft and the College created a report for individual courses. The qualitative feedback was provided from the open-ended student exam review sessions with course coordinators and the course evaluations. Feedback from faculty was also obtained via exam reviews or sessions held by the course coordinators. Faculty were able to utilise the system appropriately, once trained.

During the first block of their APPE year, students were given the opportunity to complete virtual pharmacy practice experiences in ambulatory care, and elective experiences in long-term care/geriatrics, and informatics. Students were able to access patient medical information and give pharmaceutical care plans and information using various telehealth programmes. Various tools are used to assess the APPE to ensure quality and that students were engaging in active learning. One of the assessment tools, new this year, was the requirement that pharmacy students write a onepage narrative at the end of the experience in which students describe the lessons learned and how the experience will make them better pharmacists. The pharmacy practice sites and experiences were assessed using standard site and preceptor evaluations. Efforts are underway to formally compare the pre-pandemic and pandemic experiences, innovations and outcomes.

\section{Outcomes and Recommendations}

Students struggled initially with the ExamID verification due to failure to accurately capture their facial image. This led to many inquiries to course coordinators about digital proctoring, privacy, storage, surveillance, inadequate RAM and image inversion concerns.

For the faculty, the test taker image was sometimes dark, and the reporting time increased from the initially promised 48-72 hours to three to four weeks, due to a surge in academic usage of the software. This led to delays in posting of grades, remediation opportunities and student stress. Overall, administrators, faculty and students adapted quickly.

The virtual experiential learning had a preceptor evaluation score of $4.6833 / 5$ (or $93.67 \%$ ) and a site evaluation score of $4.4833 / 5$ (or $89.67 \%$ ). The overall preceptor evaluation score for all experiences was $96.47 \%$ and that for the site evaluation was $90.52 \%$. An overall preceptor evaluation of the student was $>93 \%$. Student reflections described the impact of pharmacy and the digital industry on patient care and indicated their learning on clinical decision-making skills, effective communication, how to use technology to improve health outcomes, and how COVID-19 has helped advance the use of telehealth, thus inspiring students regarding a future career in the profession.

The faculty successfully utilised the online proctoring with artificial intelligence. This type of service can be adopted in a college of pharmacy to ensure the integrity of the exam; however, adequate instruction is needed for both faculty and students. The cost of the service and the reporting time should both be considered.

The virtual pharmacy practice experiences exposed students to new and different preceptors, different areas, and ways of pharmacy care, and will make them better pharmacists after graduation. The students' statements and overall evaluation scores show that these experiences and innovative experiential placement met both the needs of the College and students. The experience provided new areas/clerkship opportunities for the APPE students.

\section{References}

ACPE [Accreditation Council for Pharmacy Education]. (2015). Accreditation Standards And Key Elements For The Professional Program In Pharmacy Leading To The Doctor Of Pharmacy Degree: Standards 2016. Available at: https://www.acpe-accredit.org/pdf/ Standards2016FINAL.pdf

Examsoft ID verification. (2020). Preventing cheating in online courses. Available at: https://examsoft.com/solutions/id-verification

Examsoft Exam Monitor. (2020). Strengthen exam integrity with digital monitoring. Available at: https://examsoft.com/solutions/ exam-monitor 